\title{
The National Commission on Physician Payment Reform: Recalibrating Fee-for-Service and Transitioning to Fixed Payment Models
}

\author{
Mona Siddiqui, MD, MPH', Scott Joy, $\mathrm{MD}^{2}$, David Elwell, MD², and Gerard F. Anderson, $P h D^{3}$ \\ 'Division of General Internal Medicine, Johns Hopkins University School of Medicine, Baltimore, MD, USA; ${ }^{2}$ Division of General Internal \\ Medicine, University of Colorado School of Medicine, Denver, CO, USA; ${ }^{3}$ Center for Hospital Finance and Management, Johns Hopkins \\ Bloomberg School of Public Health, Baltimore, MD, USA.
}

J Gen Intern Med 29(5):700-2

DOI: $10.1007 / \mathrm{s} 11606-014-2785-1$

(c) Society of General Internal Medicine 2014

T $\mathrm{n}$ its middle six recommendations (\#4-9), the National Commission on Physician Payment Reform recognized that while it is imperative that we transition to fixed payment models, our existing fee-for-service (FFS) structure needs recalibration. FFS is currently the dominant payment model for physicians and will remain the basis for many new payment models. The Commission, therefore, enumerated transitional policies to more appropriately value and strengthen the base of the physician workforce pyramid that provides most of the evaluation and management (E\&M) services in the United States. They provide next steps for addressing imbalances in payment policies, emphasizing quality, connecting smaller practices to leverage change, and focusing first where there is greatest potential for savings. ${ }^{1}$

Among some recent steps to support primary care, the Affordable Care Act provided a $10 \%$ increase in Medicare payment rates for primary care services. The undervaluation of preventive care and care coordination has led the Centers for Medicare and Medicaid Services (CMS) to endorse additional codes for managing transitions of care from a facility-based setting to the community. Care transitions involving moderate to high complexity decisions can now be billed separately from other E\&M services. Non-face-toface communications, such as telephone and email, have been included as a component of the transitions of care codes, reflecting the significant work required in providing care management. A proposal has also been put forth by CMS to pay for non-face-to-face, complex, chronic care management services for Medicare beneficiaries who have two or more significant chronic conditions. Complex, chronic care management services would include the development and revision of a plan of care, communicating with other treating health providers, and medication management. All this will help, but continuing recalibration of relative value units (RVUs) is essential to ensure that these services are appropriately valued.

Published online February 27, 2014
With its fourth recommendation, the Commission recognized that for this recalibration to occur, annual valuations should be increased for E\&M RVUs, and that assessments for any procedural codes that may be overvalued should be frozen for a period of three years. Adjustments to RVUs by CMS must be cost neutral by law. In most years, RVU adjustments associated with E\&M services have been lower than those for procedures, although the work effort needed for many procedures diminishes as physicians gain experience. New procedures are often initially assigned a high RVU due to the related practice expense and the input cost of training. Given that the time needed to perform a procedure and the associated practice expense usually decrease with experience, the Relative Value Scale Update Committee (RUC) assessments have not necessarily reflected these adjustments. CMS has the responsibility to ensure accurate RVUs, and freezing overvalued procedure codes for three years will allow it the opportunity to use outside experts and data from additional sources to reassess values.

To further recalibrate the existing FFS structure, through its fifth recommendation the Commission endorsed equivalent payments across alternative delivery systems. The physician fee schedule payment to an office-based provider is generally higher than the payment to a provider performing a similar service in a hospital-associated outpatient department. When a service is provided in a hospital-based facility, however, Medicare makes a separate payment to the facility in addition to the physician's fee, which makes the total reimbursement for a facility service much higher than the amount the physician in the office-based setting would receive from the physician fee schedule alone. A recent report by the Medicare Payment Advisory Commission (MedPAC) identified 66 groups of services frequently performed in freestanding offices for which reducing payment differences between settings would reduce program spending and beneficiary cost sharing by $\$ 900$ million per year. The report further identified 12 groups of services performed in ambulatory surgical centers (ASCs) that would lead to $\$ 600$ million annual savings if outpatient hospital payment rates were lowered to the level of ASCs. ${ }^{2}$

Higher facility reimbursements have also contributed to physicians moving services from office to hospital settings, leading to increased total Medicare spending and higher 
beneficiary cost sharing. Many physicians are becoming salaried employees of hospitals because of this payment incentive. A survey conducted by the American College of Cardiologists found that the share of cardiologists who are employed by hospitals tripled between 2007 and 2012, from 11 percent to 35 percent. During that period, the proportion of cardiologists who work for physician-owned practices fell from 59 percent to 36 percent. $^{3}$ The Commission recognized that to promote transparency and fairness, Medicare should be neutral on rate setting and, in the absence of comparative outcome data, should not pay more simply because something is done in a hospital. Facility payments are often used to subsidize other activities for safety net institutions, and alternative funding sources may need to be explored for these organizations while monitoring access.

The recognition that FFS payments have been so wholly disconnected from outcomes led to the Commission's sixth recommendation to incorporate quality metrics into negotiated reimbursement rates within existing FFS contracts. While a transition away from FFS to fixed payment models will require aligning payment with quality metrics, it will be challenging to determine appropriate outcome measures and reach consensus across health systems and payers. Quality metrics must be instituted in a way that encourages evidence-based practice and improved health outcomes for individual patients and for patient populations.

A range of efforts are currently concentrated on incorporating quality metrics into payment models, and begs the question of which measures to choose. Should the metrics of the Medicare Physician Quality Reporting System be the standard? Should the determination of appropriate metrics be left to individual states? Should each private payer make its own assessment? Many programs are in early stages of experimentation. Evaluation of these models will provide important lessons moving forward. With the significant variation in metrics deployed by multiple payers in the marketplace, however, the administrative burden on health systems is not sustainable. Providers who will be scored on these metrics may be left questioning the relevance of these measures to the health of their patients. Provider buy-in to promote behavior change may be limited by an environment that does not have targeted, consensus driven, and patient relevant outcomes. ${ }^{4}$ Incorporating quality metrics within FFS contracts will require stakeholders to agree upon appropriate measures, harmonized across payers and systems.

Promoting quality improvement should be a high priority in all settings. The Commission's seventh recommendation acknowledged that although many recent innovations have disproportionately favored larger practices, FFS reimbursement should also encourage small practices (those having fewer than five providers) to form virtual relationships and share resources to achieve higher quality care. To support vibrant small practices that are able to embrace meaningful quality improvement strategies and participate in the innovations, the Commission recognized that virtual relationships would enable providers to retain independence, but pool their resources to provide better and more coordinated care.

Incorporating quality metrics into the existing FFS structure is a first step in moving towards fixed payment models such as bundled payments and capitation. In its eighth recommendation, the Commission acknowledged the complexity of this transition and advised that as a first step CMS target areas with the most potential for cost savings. Chronic conditions including diabetes, coronary artery disease, chronic obstructive pulmonary disease, and cerebral vascular disease represent a significant share of disease burden and have evidence-based guidelines for management. Established quality metrics may thus be more easily instituted across payers if they focus on these high cost areas that could lead to significant savings. Given the high numbers of readmissions following inpatient hospital procedures, additional cost savings could be achieved by aligning quality improvement efforts in this domain with fixed payment strategies.

Although transitioning to fixed payment models has significant potential for cost savings, the Commission acknowledged in its ninth recommendation that monitoring the impact of these payment models on physician access will be essential. To ensure that providers are not "cherry picking" patients that are low complexity and low cost, adequate risk adjustment will be critical. Moreover, continuing evaluation and monitoring efforts will be required to be certain that services are not inappropriately withheld, as this often has the greatest adverse impact on those at the lowest end of the socioeconomic spectrum with historically worse health outcomes.

Transitioning from a system based solely on FFS to one that embraces fixed payments is a paradigm shift that has already begun. This evolution will not be easy. The Commission appreciated this in its considered approach to overhauling the current underlying valuation methodology, linking FFS to quality metrics, and focusing on high yield areas for fixed payment models. This stepwise approach will be critical to enabling an improved payment system, mindful of cost, and driven by outcomes.

Conflict of Interest: The authors declare that they do not have a conflict of interest.

Corresponding Author: Mona Siddiqui, MD, MPH; Division of General Internal MedicineJohns Hopkins University School of Medicine, Hampton House, Room 626, 624 North Broadway, Baltimore, MD 21205-1901, USA (e-mail: msiddiq2@jhmi.edu). 


\section{REFERENCES}

1. http://physicianpaymentcommission.org/wp-content/uploads/2013/ 03/physician_payment_report.pdf Accessed 10/05/2013.

2. Medicare Payment Advisory Commission, Report to the Congress: Medicare and the Health Care Delivery System. (Washington: MedPAC, June 2013), chap 2.
3. American College of Cardiology. New American College of Cardiology Practice Census Shows Continued Trend Towards Hospital Integration. http: / /www.cardiosource.org/News-Media/Media-Center/News-Releases/2012/09/Leg-Conf.aspx. Accessed 10/08/2013.

4. Berenson RA, Kay DR. Grading a Physician's Value-The Misapplication of Performance Measurement. N Eng J Med. DOI: 10.1056/ NEJMp1312287. 